\title{
El papel del periodista en la información política electoral y uso de Twitter en la difusión de contenidos
} The role of the journalist in political election reporting and the use of Twitter in the dissemination of content

Lorena Parra Gómez, Universidad de Málaga - parragomezlorena@gmail.com

Abstract

The present study focus in the role of the journalist in the elaboration of political information during elections and how they use new technologies for contents diffusions. We want to clarify how nationals newspaper journalist use Twitter, with speciality in politics issues and being authors in the elaboration of news during several electoral campaigns, general or European. Regularity in the use of this tool; types of contents that they share; prototypes of users with whom they interact; and relevance or credibility that they bestow about the political parties share in the social networks, are some of the aspects to delimitate.

Keywords

Political journalism, social networks, politics, communication, twitter.

Resumen

El presente estudio se centra en el papel del periodista en el tratamiento de la información política electoral y en cómo estos hacen uso de las nuevas tecnologías para la difusión de contenidos. Se busca esclarecer cómo hacen uso de Twitter los redactores de periódicos de ámbito nacional especializados en información política y que han participado en la elaboración de noticias durante diversas campañas electorales, tanto generales como europeas. Regularidad en el uso de esta herramienta; tipo de contenidos que comparten; prototipo de usuarios con los que interactúan; y relevancia o credibilidad otorgada a la información compartida por los organismos políticos en la redes sociales, son algunos de los aspectos a delimitar.

Palabras clave

Periodismo político, redes sociales, política, comunicación, twitter.

1. Introducción; 2. Marco de la cuestión; 3. Objetivos y metodología; 4. Resultados; 5. Conclusiones; 6. Bibliografía. 


\section{Introducción}

Los medios son la principal fuente de información sobre la política y el principal canal de comunicación entre los actores políticos y los ciudadanos (Strömbäck, 2008). Según el V Estudio anual de redes sociales del IAB (Interactive Advertising Bureau), en España el 82 \% de los internautas son usuarios de las redes sociales (14 millones) y usan un promedio de 3 redes sociales por persona. Internet se ha convertido en un medio al que poder acceder desde cualquier lugar y a cualquier contenido.

Los medios tradicionales llegan a un $92 \%$ de los usuarios de internet que consumen noticias según el estudio realizado por Reuters Institute Digital News Report 2014, en colaboración con la Universidad de Navarra. Las redes sociales se alzan como la herramienta elegida por un $38 \%$ de los usuarios para conocer las últimas noticias, mientras que un $46 \%$ accede a estos directamente vía sitio web o aplicación del medio en cuestión. Además especifica cómo los usuarios españoles conceden más importancia a la firma del periodista que a la marca de la publicación a la hora de otorgarles credibilidad, considerando "muy importante" para el $60 \%$ de estos la firma del autor y un $55 \%$ la marca o cabecera del medio, aumentando la confianza en el autor conforme aumenta la edad del encuestado.

Basándonos en estos datos sobre las tendencias de consumo de información a través de internet y uso de redes sociales, y teniendo presente la importancia del periodista como mediador en la elaboración de noticias de información política y su influencia como generadores de opinión, este estudio se centrará en delimitar cual es el papel del periodista en la red social twitter y el modo en que utilizan esta herramienta para difundir contenidos.

Desde el punto de vista metodológico, nos apoyamos en la técnica de la entrevista en profundidad realizada a 6 periodistas especializados en información política que publican a diario en prensa escrita de ámbito nacional tales como El País, El Mundo, La Razón, ABC y La Vanguardia, y que están activos en la red social Twitter. La finalidad es desentrañar cómo esta herramienta es usada por los profesionales del medio y se ha convertido en una especie de extensión de la redacción vía los perfiles personales de sus redactores, los cuales al retuitear noticias del medio, acercan a nuevos usuarios a los contenidos.

\section{Marco de la cuestión}

Las modificaciones en las formas de comunicación que han ido surgiendo durante las últimas décadas, han supuesto la transformación de las relaciones entre emisores, medios y receptores, bosquejándose un panorama en el que se han sustituido los debates públicos en las plazas por los medios de comunicación (y hoy día también las redes sociales), como único escenario en el cual se debate la información política.

Los medios de comunicación han jugado un papel decisivo en la evolución de los procesos de comunicación política, convirtiéndose la televisión e Internet, en los ejes centrales en torno a los cuales gira todo intento de comunicación entre gobernantes y ciudadanos. Ignacio Ramonet considera que la televisión impone la necesidad de obtener una mayor expresividad teatral, marcando este hecho un antes y un después. Asimismo M. Alonso explica que $<<$ los políticos viven de y para los medios, y su agenda viene determinada por las exigencias que tales medios plantean>> (Muñoz Alonso, 2008:37-42).

Vivimos en una sociedad, en la que las posibilidades de comunicación se han visto enormemente incrementadas, en la que ya no son tan necesarios los medios de comunicación para llegar a públicos masivos (Sey y Castells, 2006:440-463).

El incremento de las nuevas tecnologías y el crecimiento en los últimos años de usuarios de Internet han supuesto el acceso de millones de personas a una vía de interacción y comunicación abierta entre medios, usuarios y políticos, en la que los recursos digitales han impulsado cambios en las rutinas de trabajo en todos los ámbitos y profesiones.

La población accede como, cuando y donde quiere a la información, por lo que se han modificado los escenarios que venían siendo los pilares de comunicación entre políticos y electorado. Fenómenos como la "globallización" o "americanización" han tenido repercusión en el ámbito de la comunicación e información política, transformándose las técnicas, herramientas y estrategias que utilizan los partidos durante las campañas, y modificando la manera en la que deben gestionar sus recursos los profesionales del periodismo.

La "especialización de los profesionales de la comunicación" y las nuevas tecnologías y redes sociales, delimitan un escenario en el que los nuevos medios y las redes sociales suponen el eje en torno al cual se estructuran las estrategias de comunicación de los partidos políticos, cuya agenda marca casi a diario la agenda mediática y periodística, y en base a la cual se generan los contenidos que serán consumidos por los usuarios.

La digitalización de los medios y la gran intrusión de las redes sociales condicionan la forma en la que hacer periodismo. Toda forma de democracia requiere medios de comunicación política, tales como reuniones cara a cara, periódicos, radios, televisi para informar a los ciudadanos, respaldar a los gobiernos al dar explicaciones y facilitar la discusión pública. Sólo difieren en su alcance y capacidad de emisión (Loader, B. D. Y Mercea, D. 2012).

La digitalización y la red 2.0, han otorgado nuevas herramientas, no sólo a políticos y periodistas, sino también al público en general que puede convertirse en productor y difusor de mensajes gracias a las herramientas digitales (Allan, 2013), constituyendo una nueva dimensión del público.

Para que exista una verdadera comunicación tiene que existir esa posibilidad de respuesta (Carrascosa, 1992). La Red se ha convertido en la nueva esfera en la que medios, usuarios y políticos acuden para crear y obtener información y en definitiva para comunicarse y generar feedback. 


\section{Objetivos y metodología}

Varios son los estudios realizados hasta el momento sobre la relación de los periodistas con las nuevas tecnologías y en concreto sobre redes sociales. Especial es el caso de Twitter, que se ha convertido para muchos usuarios en una herramienta de acceso rápido a titulares donde prevalece la brevedad e inmediatez.

La audiencia web prefiere los titulares y prioriza el texto sobre lo multimedia, más de la mitad del público online español mira portadas y el $40 \%$ lee noticias y artículos. Twitter y Facebook ya superan a los diarios impresos como fuente informativa incluso entre los usuarios de una franja de edad entre 35 y 44 años. ${ }^{1}$

Como muestra el estudio "Join the Conversation: How spanish journalism are using twitter"2, en el que tras realizar una encuesta en profundidad a 50 periodistas españoles, buscan esclarecer cual es el uso profesional que hacen de las redes sociales así como analizar su percepción de las mismas y expectativas, el 95\% utilizaban esta herramienta como medio de distribución de información, así como un 82\% lo hacían para compartir información precedente de los propios medios.

Datos aportados por el V Estudio Anual de Redes Sociales (IAB), muestran como Facebook sigue siendo la red social más conocida y con mayor penetración (96\%), seguida de Youtube $(66 \%)$ y de Twitter $(56 \%)$, siendo Facebook (7 días a la semana) y Twitter (4,9 días) las primeras redes en cuanto a la frecuencia de uso, seguidas de Instagram (4,4 días).

Tras los datos analizados hasta el momento, y observando la evolución de las redes sociales en la difusión y creación de información, es interesante abordar el objetivo del estudio para realizar un acercamiento a la visión del periodista de información política en España y su relación con las nuevas tecnologías y uso de Twitter como plataforma para la difusión de contenidos.

El presente trabajo se basa metodológicamente en una serie de entrevistas en profundidad realizada a 6 periodistas de reconocida trayectoria periodística, especializados en información política que publican a diario en prensa escrita de ámbito nacional tales como El País, El Mundo, La Razón, ABC y La Vanguardia, y que están activos en la red social Twitter.

Según Orihuela, Twitter "ha cambiado la red y ha completado el giro social que iniciaron los blogs a finales de los años 90" (2011:21). Una red de microblogging que favorece el feedback entre usuarios y que va "más allá de la tecnología, son las personas que lo utilizan, los mensajes que comparten, las conversaciones que se mantienen y las relaciones que se establecen entre los usuarios" (Orihuela, 2011:31).

Debemos reseñar que en un principio el estudio estaba dirigido a obtener datos de 10 periodistas (2 por medio elegido) y se contactó desde el inicio con un total de 19 periodistas, de los cuales 6 accedieron al responder y completaron la entrevista; 7 contestaron que accederían pero no la completaron; 4 no respondieron; y 2 afirmaron que no era su especialidad en el momento y preferían declinar hacerla.

La entrevista estuvo compuesta por preguntas de carácter abierto, complementadas con otras de tipo cuestionario en las que entre otros aspectos se buscaba la valoración numérica de aspectos como la credibilidad en las fuentes. Esta estructuración de la entrevista alrededor de elementos cuantitativos y cualitativos, vino motivada por la necesidad de obtener información completa y no limitada sobre la percepción que tienen estos profesionales periodísticos sobre Twitter como herramienta en el desarrollo diario de su trabajo y observar si ésta se ha convertido en una especie de extensión de la redacción vía los perfiles personales de sus redactores, quienes a su vez pueden acercar nuevos usuarios a los contenidos.

Twitter permite difundir información de forma exponencial de modo que cualquier información aquí compartida puede propagarse fácilmente a través de la red, y esto unido a la figura del periodista como perfil generador de opinión pública y creador de contenidos en época electoral, supone un ámbito de estudio que merece ser tenido en cuenta, y es el que abordamos en este estudio.

\section{Resultados}

Según datos del estudio "Join the Conversation: How spanish journalism are using twitter", el 95\% de los periodistas usan asiduamente Twitter como instrumento de publicación y distribución de información, y tan sólo un 25\% asegura utilizarla para realizar periodismo de investigación. En este epígrafe cuestiones como estas y otras son las que buscamos esclarecer, haciendo hincapié en el tipo de información que difunden; perfiles con los que tienen un mayor o menor feedback; evaluación de perfiles políticos e institucionales; y valoración del este espacio en función de su efectividad, etc.

Casero-Ripollés señala que políticos y periodistas definen conjuntamente la realidad política que llega a los ciudadanos y establecen así los contenidos y límites del debate público ${ }^{3}$. Entonces ¿condicionan los partidos la agenda mediática? Según las respuestas de los 6 entrevistados al respecto, 3 respondieron Si y 3 No. Al tratarse de una respuesta abierta debemos reseñar que de los 6 entrevistados 3 especificaron que condicione o no la agenda mediática, se trata de información que el ciudadano debe y tiene derecho a conocer ya que son sus representantes, como sería el caso de las sesiones del Congreso para informar sobre la tramitación de leyes.

Por su parte 2 de los entrevistados subrayaron que estas citas diarias con los partidos o las instituciones forman parte de su trabajo y no por ello tienen que servir para meramente reproducir declaraciones, sino que puede ser la base de elaboración de un artículo bien

\footnotetext{
${ }_{1}^{1}$ Reuters Institute Digital News Report 2014: Spain http://www.unav.edu/web/digital-studies/news

2 "Join the Conversation: How spanish journalism are using twitter" LABáPART, (UC3M)

http://www.pilarcarrera.es/jointheconversation.html

${ }^{3}$ CASERO-RIPOLLÉS, Andreu. 2012. Periodismo político en España: concepciones, tensiones y elecciones. CAL, Cuadernos Artesanos de Latina (33),19-46.
} 
contrastado, a lo que añadieron que es precisamente en cualquier rueda de prensa y mediante las preguntas, el momento en el que el periodista establece la agenda.

Cabe señalar que, uno de los participantes aportó una afirmación en base a la cual diferenciaba entre el medio en papel o soporte web, y aseguraba que era precisamente en la web donde se percibía un mayor condicionamiento de la agenda, mientras que el papel estaba en proceso de destierro de ese tipo de noticias. ¿Estamos quizás ante una nueva forma de hacer periodismo en papel en el que las instituciones o partidos no marquen los tiempos? ¿Es la publicación vía soporte web de menor calidad cuando es a la que más usuarios acceden? 0 ¿es esto fruto de las rutinas de los usuarios basadas en la obtención de información rápida las que están proporcionando nuevos estilos de periodismo para la red?

Tras comprobar la discrepancia de opiniones sobre el condicionamiento de la agenda mediática, abordaremos si estas se fundamentan en la manera en la que dichos profesionales acceden a la información a través de distintas fuentes y la validez que les otorgan.

Tabla 1. Frecuencia con la que reciben información a través de las distintas herramientas

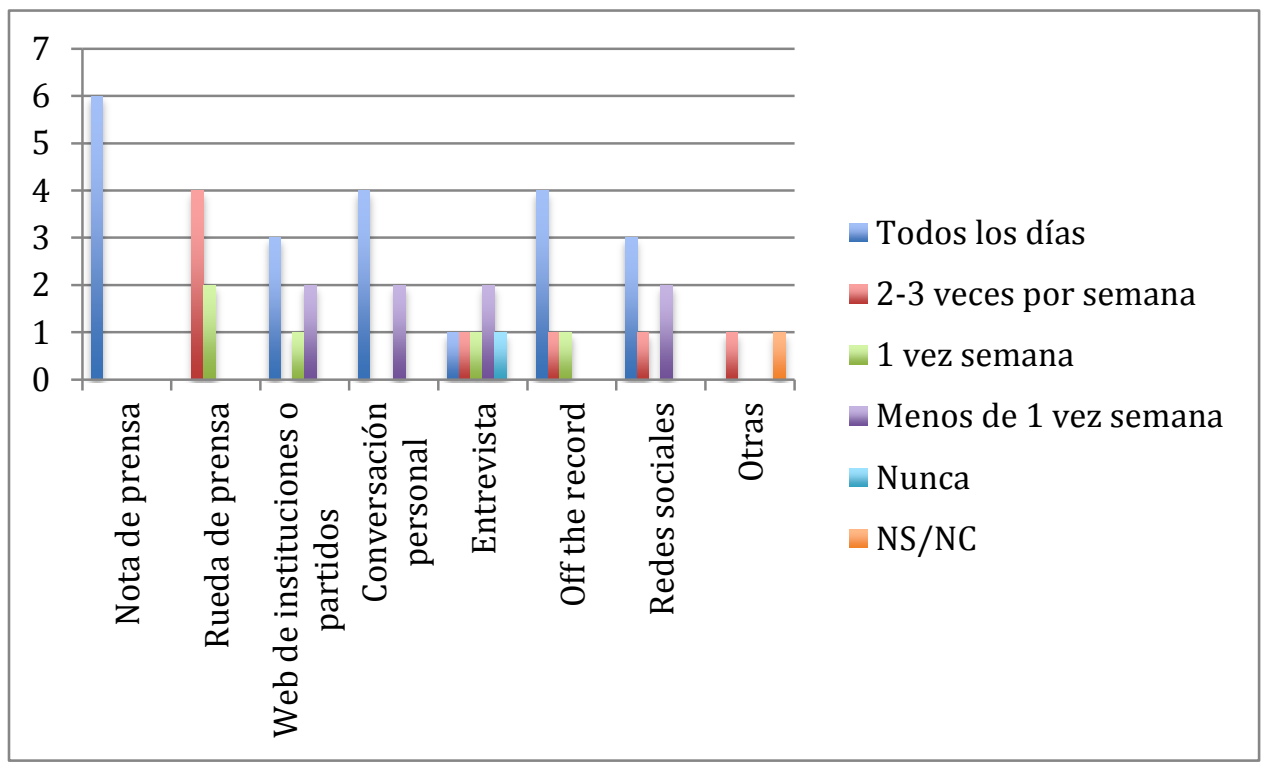

Tabla 2. Credibilidad que le otorgan (siendo 1 la mínima puntuación y 5 la máxima)

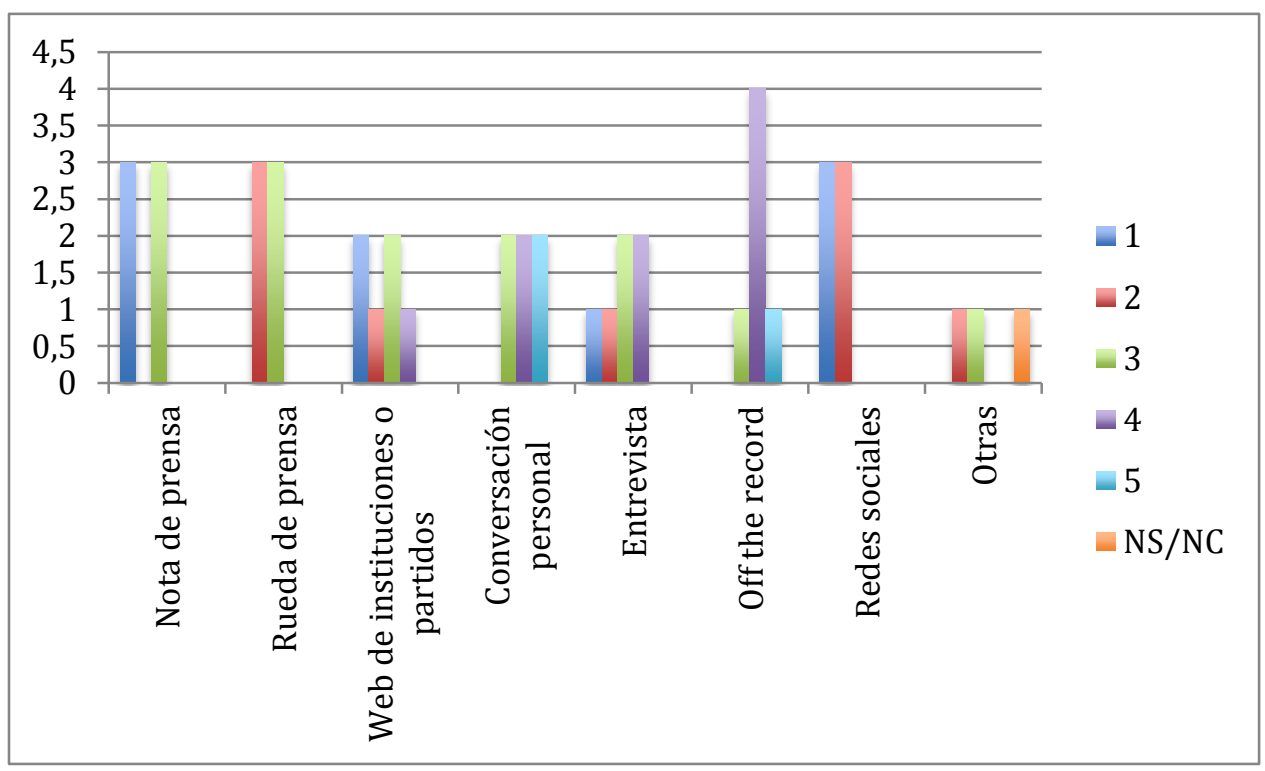


- $\quad$ Nota de prensa: recibida por el $100 \%$ de los entrevistados, cuenta con una valoración media de 2 puntos sobre 5 en credibilidad.

- Rueda de prensa: un 66\% acude 2-3 veces a la semana y un 33\% 1 vez a la semana. Valoración media de 2,5 puntos sobre 5.

- Web de instituciones o partidos: el 50\% las utiliza todos los días; un 33\% 1 vez a la semana; $16 \%$ menos de 1 vez a la semana. Valoración media de 2,33 sobre 5.

- Conversación personal: el $66 \%$ todos los días; $33 \%$ menos de 1 vez a la semana. Valoración media de 4 sobre 5.

- Entrevista: $33 \%$ menos de una vez a la semana; $16 \%$ 2-3 veces a la semana; $16 \%$ una vez a la semana; $16 \%$ todos los días. Valoración media de 4 sobre 5.

- $\quad$ Off the record: $66 \%$ todos los días; $16 \%$ 2-3 veces a la semana; $16 \% 1$ vez a la semana. Valoración media de 3,4 sobre 5.

- Redes sociales: 50\% todos los días; 33\% menos de una vez a la semana; $16 \%$ 2-3 veces a la semana. Valoración media de 1,4 sobre 5 .

Con estos resultados observamos como la "conversación personal" y "off the record" son las herramientas mejor valoradas en cuanto a credibilidad por los propios periodistas, los cuales en su mayoría (en ambos caso $66 \%$ ) utilizan estas fuentes a diario. Todos coinciden en señalar que reciben notas de prensa diariamente, pero son estas, junto con las redes sociales, las que menor credibilidad les proporcionan (2 y 1,4 puntos respectivamente). La entrevista es la herramienta menos utilizada, aunque la tercera mejor valorada en cuanto a credibilidad.

Como se distingue, no son grandes las diferencias observadas en relación a la utilización de estas herramientas, de ahí la necesidad de introducir preguntas abiertas en las que los participantes, puedan aportar una mayor cantidad de información y esclarecer el por qué del uso de ciertas fuentes en detrimento de otras, como es el caso de la utilización o no de los recursos web ofrecidos por las instituciones o partidos políticos o de sus perfiles en las redes sociales.

Ya hemos indicado que la valoración más baja en cuanto a credibilidad ha sido otorgada a las redes sociales, estas y los recursos web de las instituciones no aprobarían en cuanto a credibilidad se refiere. Aquí separaremos dos cuestiones:

1. Sobre la utilización o no de recursos web ofrecidos por las instituciones o partidos políticos.

2. Aplicado al manejo o no de las redes sociales desde el punto profesional.

En la primera cuestión 3 de los periodistas afirmaron acceder a ellos sólo para conocer la información oficial y convocatorias, así como acceder a archivos oficiales; 2 aseguran seguir estos perfiles pero en ningún caso como fuente de información principal y sin contrastar; 1 señaló que a veces si utiliza estos recursos pero no añadió información. Un elemento en común en 5 de las 6 respuestas es la aclaración de que estos perfiles pueden aportar información pero no es considerada muy útil, salvo en el caso de algún tipo de comunicado oficial realizado a través de estas.

En cuanto a la segunda cuestión, y relacionada con el manejo o no de las redes sociales desde un punto de vista profesional, 5 de los 6 entrevistados aseguran utilizar las redes sociales de forma mixta o exclusivamente profesional. Discusión de textos propios; difusión de noticias de su publicación; subir información de terceros que consideran interesante; consulta e intercambio de información; etc. Sólo 1 de los profesionales afirmó no usarlas en el ejercicio del periodismo; aunque si en ocasiones para la fijación de una posición rápida sobre algunos aspectos.

Nos encontramos pues en una sociedad marcada por las nuevas tecnologías, en la que pese a la poca valoración inicial y precaución en cuanto al uso de informaciones obtenidas a través de las redes sociales, la mayoría de estos profesionales del periodismo político han aceptado estos nuevos soportes como una parte complementaria a los medios tradicionales.

Por su brevedad e inmediatez, Twitter se ha caracterizado por ser una plataforma a la que acuden muchos usuarios para estar informados, frases cortas; enlaces a web y/o medios de comunicación donde poder ampliar las noticias; posibilidad de interacción y contacto directo usuario-político-periodista, hacen de esta red social una plataforma de comunicación adaptada a las nuevas demandas de los usuarios. Las reglas del juego han cambiado, ahora el concepto clave es la conversación. El votante ya no sólo escucha, sino que también habla (Ureña, 2011:31).

Una vez conocidos los principales hábitos y percepción sobre las redes sociales por parte de los entrevistados, nos centraremos en el uso que estos hacen de este social network; sus opiniones en relación a las aportaciones a la profesión; frecuencia de uso; e información compartida; todo ello nos permitirá entender mejor el comportamiento de estos profesionales en el ámbito de las redes sociales, así como delimitar qué tipos y cómo se difunden los contenidos a través de estas.

Los entrevistados presentan opiniones contrarias en cuando a si Twitter es imprescindible o no, la mitad si lo considera imprescindible mientras que la otra mitad no. Sin embargo, y considerándolo o no, todos apuntan a que lo que si es imprescindible es saber filtrar que contenidos que son erróneos o no rigurosos.

En cuanto a aspectos positivos, los participantes destacaron la rapidez, la concisión, el volumen de información y medición de la repercusión entre los lectores. Uno de ellos apuntó que sirve de inspiración y aprendizaje en cuanto a la forma en que periódicos de renombre extranjeros publican las noticias y añadió que si se ha convertido en un medio imprescindible para los medios en la difusión de contenidos.

Por su parte, los aspectos negativos señalados por los periodistas son el peligro de no saber filtrar los contenidos y la creación de vicios que supone el quedarse sólo con las opiniones vertidas en 140 caracteres. Por último todos los periodistas afirmaron no considerar que esta red haya aportado gran cosa a la información política en concreto. 
Tras conocer su opinión acerca de las aportaciones de esta red para el periodismo político, nos centramos en esbozar cual es la frecuencia de uso y los tipos de contenidos que comparten en sus perfiles personales estos profesionales, para de esta manera ir perfilando la forma en la que se sirven de este espacio.

Tabla 3. Frecuencia de uso de Twitter

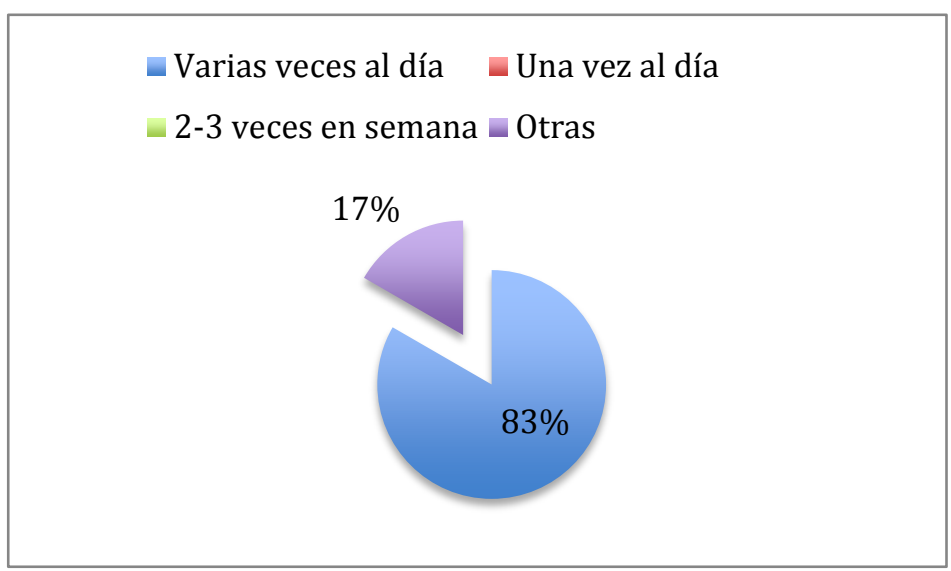

Tal y como se observa en la gráfica, la mayoría (83\%) usan esta red varias veces al día y sólo uno de los consultados señaló la opción otros $(17 \%)$, datos que apuntan a señalar que dicha red ya forma parte de su rutina diaria de estos profesionales.

En cuanto al tipo de contenidos que comparten en Twitter y en alusión a si realizan tuits solo del medio o especialidad o enlaces de otros medios los resultados son los siguientes:

- De los 6 periodistas $4(66 \%)$ comparten noticias del medios y/especialidad así como pueden incluir noticias de otros medios 0 informaciones que les parezcan interesantes. De estos uno especificó que aunque pueda incluir noticias de otros medios, intenta evitar difundir información de la competencia directa al igual que no recomendaría la compra de otro diario que no fuese el suyo.

- Sólo 2 de los 6 (33\%) afirmó retuitear noticias de las webs para el medio en que trabaja y no de otro. De los que 1 afirmó compartir informaciones de algunas instituciones.

A la vista de estos resultados, y en relación a la pregunta planteada al principio de la investigación sobre Twitter como herramienta en el desarrollo diario de su trabajo y el modo en que se ha convertido en una especie de extensión de la redacción vía los perfiles personales de sus redactores, podríamos aseverar que en cierto modo esta afirmación es correcta dado que 100\% de los participantes afirman compartir información de su medio.

Es cierto que no debemos obviar que el $66 \%$ de los aquí consultados afirmaron enlazar informaciones de otros medios o especialidades si las consideraban interesantes, pero debemos reseñar también que uno de ellos delimitó que intenta no difundir informaciones directamente competitivas y dos más declinaron compartir información de medios que no fuera el suyo. Por lo que estaríamos hablando que casi un $50 \%$ de los participantes no incluye nunca o casi nunca información que no sea de su medio.

De este modo, podemos dibujar un panorama periodístico dentro de la especialidad de información política, en la que sus protagonistas usan Twitter en su mayoría a diario (83\%) y comparten en su totalidad información relacionada directamente con su trabajo (además de otras), por lo podríamos decir que las informaciones elaboradas en las redacciones van más allá de las ediciones en papel, digitales, 0 páginas oficiales en redes sociales, y se adentran en los perfiles personales de quienes forman parte de la plantilla y publicitan su trabajo y el de sus compañeros.

Tras observar la relación directa existente entre los contenidos compartidos por nuestros protagonistas y las publicaciones de sus medios, nos centraremos ahora en esclarecer, si dada la naturaleza de esta relación periodista-información-medio, desde las redacciones existe algún tipo de directriz o manual de estilo para el uso de las redes sociales, y en su defecto preguntaremos también si en algún momento especifican en su perfil que sus opiniones son de tipo personal.

No debemos olvidar que los medios han tenido que adaptarse a las nuevas circunstancias y modos de consumo de información surgidos tras los enormes avances tecnológicos y que han supuesto que la mayoría de usuarios de internet puedan acceder como y cuando quieren, desde el dispositivo elegido (tablets, teléfonos móviles, etc) a todo tipo de información.

Es por ello el interés por averiguar si desde las publicaciones se han adaptado también sus manuales y añadido el uso de las redes sociales como espacios en los que se comparte gran parte de las noticias publicadas en los medios, y en gran medida por sus mismos periodistas. Todo esto sin obviar que se trata de un tema muy delicado dados los frágiles límites que delimitan la sugerencia, de la intromisión en la vida personal de sus trabajadores, ya que no podemos olvidar que no dejan de ser perfiles personales.

Ante esta pregunta sólo uno de los profesionales entrevistados afirmó tener unas guías o directrices ante el uso de las redes sociales, tales como no colgar información que se encuentre en cerrado en la página web, o no olvidar nunca que si especifican en su perfil que 
pertenecen a ese medio, forman parte de la imagen de este. El resto, un $83 \%$, aseguró que no existe ningún tipo de directriz, más que la responsabilidad, la prudencia o el uso del sentido común. De estos 5 periodistas, 2 afirmaron que hubo un intento en sus medios por realizar algún de regulación pero frente a las protestas de los redactores no se terminó de definir, y uno de ellos alegó a que lo que existe actualmente en su medio sería más bien una "autoregulación" en la que todos saben que no se debe atacar a su medio/empresa, así como no desvelar noticias exclusivas antes de que se publiquen. Otro de los periodistas que afirmó no tener ningún tipo de directriz desde su medio para el uso de las redes sociales, afirmó que sería interesante que existiese, enfocada al buen uso y potenciamiento de esta plataforma.

Al entrar en perfiles de muchos profesionales del medio, se observa como algunos muestran frases como la siguiente "aquí, sólo opiniones personales". Ante esta característica, quisimos preguntar si nuestros protagonistas tenían algún tipo de mensaje similar y sus opiniones sobre esta cuestión.

Sólo 2 de los 6 participantes especifican en su perfil que sus opiniones son personales, de hecho uno de ellos comparte en este su perfil personal y no el profesional para distanciarse y dejar claro que se trata de un perfil personal. Si bien es cierto que 4 de los 6 da por entendido que al tratarse de un perfil personal no tienen por qué especificar nada, uno de ello analiza el sentido que tiene que algunos compañeros suyos lo adviertan, como un medio de prevención.

Una vez analizados aspectos como mediatización de la agenda; tipos de información y credibilidad otorgada según la fuente; uso de recursos vía web o redes sociales y utilización de forma profesional; aportación de Twitter al periodismo político; frecuencia de uso de esta plataforma o tipos de contenidos compartidos; nos centraremos en una de las características más destacables de esta red social, la posibilidad de feedback y comunicación directa entre usuarios de diversa índole.

Tabla 4. Generación de feedback en función del perfil del usuario (siendo 1 la mínima interacción y 5 la máxima)

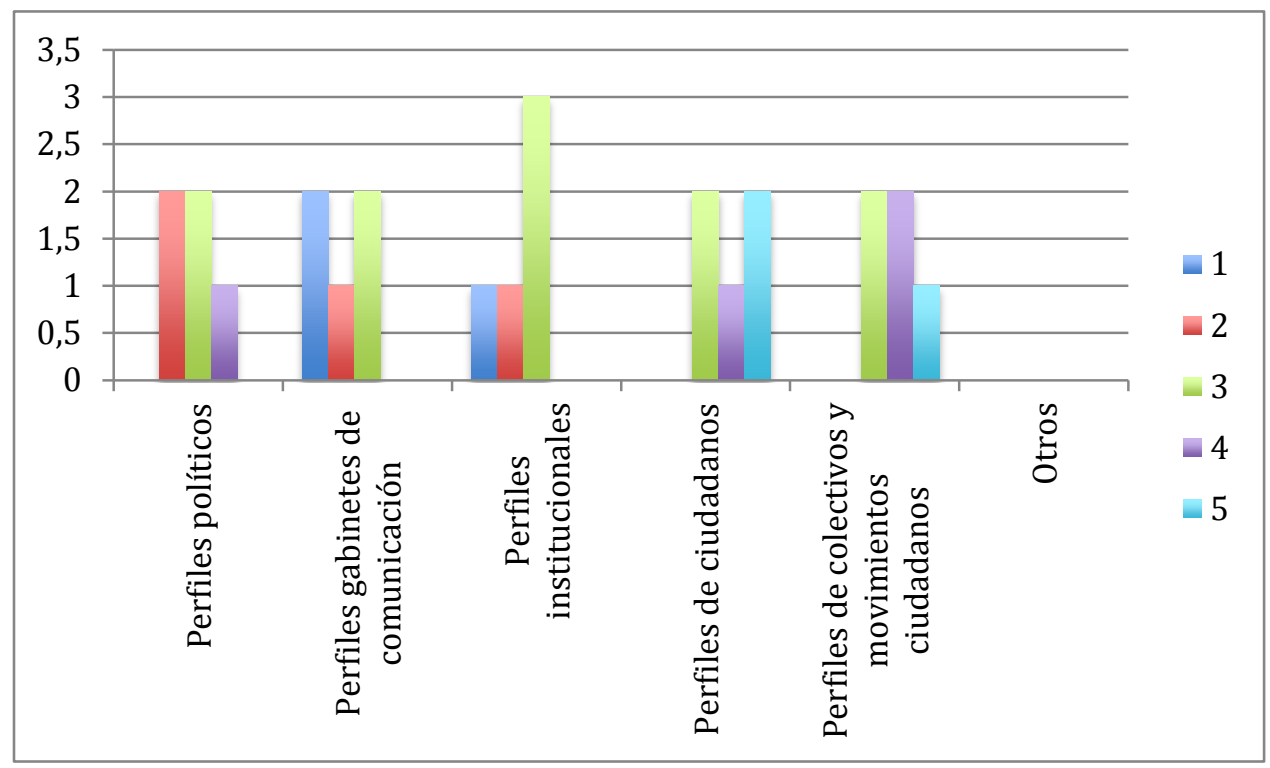

Los resultados aportados sobre interacción en función del tipo de perfil nos indican que el mayor feedback se produce con los perfiles de usuarios, con una puntuación de 4 sobre 5 , seguido de los perfiles de colectivos o movimientos ciudadanos ( 3 sobre 5 ); en tercer lugar nos encontraríamos con los perfiles relativos al marco institucional con una media de 2,6. En cuanto a los que menos interacción provocan nos encontraríamos con los perfiles de los políticos (2 sobre 5), y el perfil menos participativo sería el de tipo institucional. Debemos apuntar que en esta pregunta uno de los participantes no puntuó según el tipo de perfil si no que señalo dos con los que se genera mayor feedback de ahí la media realizada sobre 5. En cuanto a sus anotaciones es importante señalar que sus dos perfiles marcados como los de mayor flujo de comunicación coincide con los dos que obtienen una mayor puntuación por parte del resto de entrevistados, esto es perfiles de ciudadanos y perfiles de colectivos o movimientos ciudadanos.

A la vista de estos datos, en los que los usuarios, bien individualmente o a través de perfiles de colectivos, son los generadores de mayor feedback, y una vez delimitado el tipo de contenidos y regularidad con la que usan Twitter nuestros protagonistas, nos dirigimos a ellos para saber si utilizan esta web para solicitar información y hacer preguntas a los usuarios para después trasladarlas a los políticos, haciéndolos participes a través de sus perfiles.

En su totalidad negaron hacer preguntas a los usuarios para después trasladarlas a los políticos, y sólo uno de ellos afirmó usar Twitter como herramienta para solicitar información y otro mostró tener interés en conocer que temas si gustaría a los usuarios encontrar en su medio y no hallan. Uno de los periodistas añadió que precisamente una de las ventajas de esta red, es que permite que sean los mismos usuarios quienes se pongan en contacto directo con los políticos.

Por último, otro de los entrevistados añadió que se puede correr el riesgo de pensar que toda la sociedad está en Twitter y no es así, es más añadió que periodistas que se apoyan mucho en esta corren el riesgo de recibir presiones sobre el qué y el cómo, pasando a ser más importante la pregunta que la respuesta convirtiéndolo en una forma de anti-periodismo. 
Tras esta pregunta, buscamos delimitar cuáles son los perfiles en el caso de obtener algún tipo de información a través de las redes sociales, a los que estos periodistas especializados en información política prestan más atención. No podemos olvidar que estos profesionales elaboran la información que será consumida por los usuarios y a través de las que la mayoría se informan de qué, cómo y cuándo acerca de sus representantes, por tanto, será interesante observar si entre periodistas y políticos se produce interacción a través de esta nueva plataforma caracterizada precisamente por el feedback entre usuarios.

Tabla 5. Atención prestada según perfiles para obtención de información

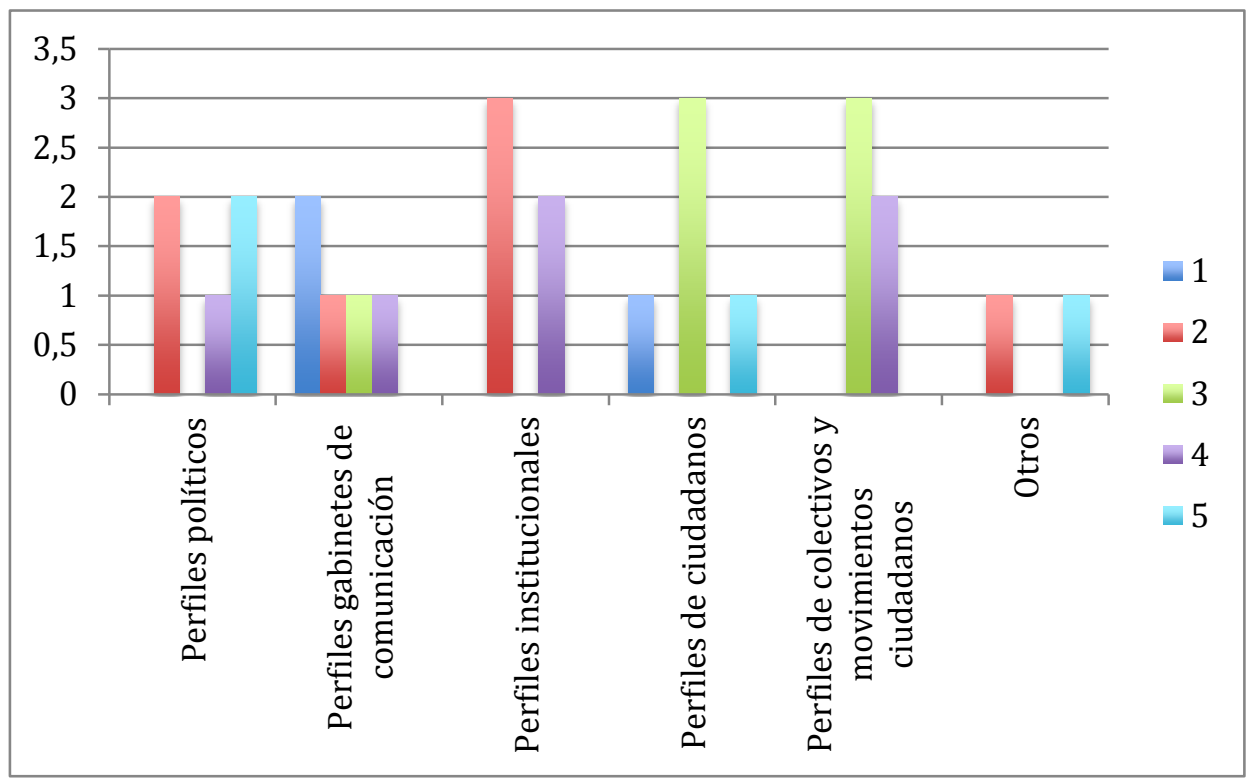

Según las puntuaciones aportadas, el tipo de perfil al que prestan más información es el político (3,6 sobre 5), en segundo lugar se encontrarían los perfiles ciudadanos ( 3 de 5 ) y en tercer lugar los de colectivos y movimientos ciudadanos. Los menos seguidos son los gabinetes de comunicación (2,2 de 5) y los institucionales (2 de 5). En este apartado un participante contestó de forma incorrecta, sin valorar todos los perfiles, aunque si señaló que los dos a los que presta más atención serían los perfiles ciudadanos y otros, refiriéndose a los perfiles de periodistas. Hemos de señalar que un punto a incluir podría haberse incluido y debería incluirse en futuras investigaciones.

Si ponemos el punto de mira en el perfil al que prestan mayor atención según los datos facilitados, el perfil político, buscamos ahora complementar esta información para seguir definiendo el papel que juega el periodista en la información política y electoral. Para ello consideraremos ahora si durante las elecciones estos profesionales aumentan o no su actividad y les preguntaremos si interactúan o no con estos.

Tabla 6. Aumento o no de actividad en redes sociales durante la campaña electoral e interacción con políticos a través de dichas redes

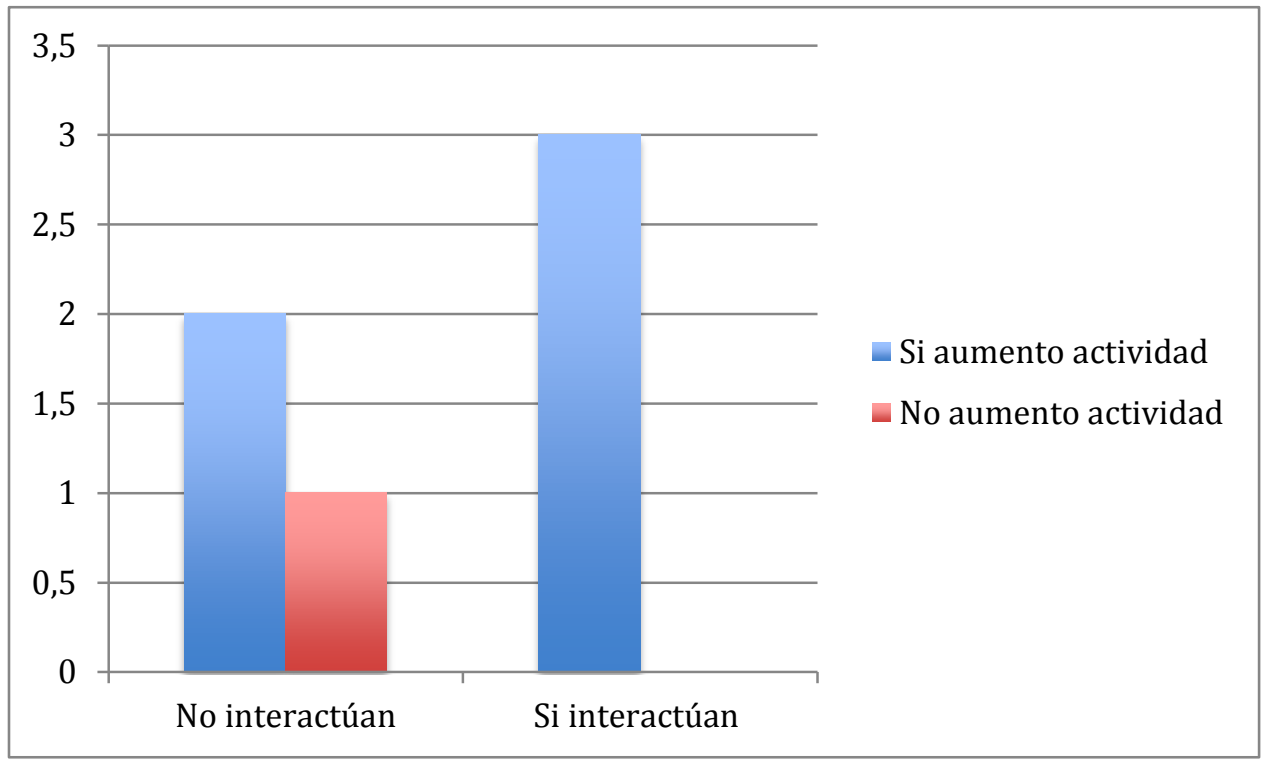


De los 6 entrevistados, 5 (83\%) admiten aumentar su actividad durante las campañas electorales, mientras que 1 (16\%) asegura no aumentar dicha actividad ante estos periodos. Del total de los 5 periodistas que aumentan su actividad un total de 3 interacciona con los políticos a través de las redes sociales, mientras que los otros 2 que alegaron aumentar su actividad más el que no, aseveran no interaccionar con estos perfiles, aún en campaña electoral.

Llegados a este punto, quisimos abordar la opinión que tienen, como profesionales de la información política y conocedores de los perfiles de los representante políticos, estos periodistas sobre el uso que hacen los políticos de Twitter $u$ otras redes sociales.

Los preguntados coinciden en señalar que por lo general los políticos andan muy desorientados en cuanto a la utilización de las redes sociales y en su mayoría señalan también, cómo uno de los problemas que observan es que en su mayoría no son los propios políticos, sino su equipo quienes suelen llevar los perfiles, algo negativo desde su punto de vista. Otros aspectos y respuestas aportadas sobre el uso de Twitter por parte de los políticos son las siguientes:

- "Hay quienes son demasiado atrevidos y generan demasiadas e innecesarias polémicas. Creo que hay quienes no son conscientes todavía de la repercusión de las redes sociales"

- "Todos los políticos lo utilizan como un vehículo más para difundir propaganda, pero no son conscientes de algunos riesgos. Creo que muchas veces tienen más que perder que que ganar si se arriesgan demasiado. En cualquier caso, como lo hace un sector de la sociedad muy determinado (principalmente vinculado a los medios de comunicación), los políticos y partidos sobrevaloran la influencia de Twitter y Facebook"

- $\quad$ "Lo utilizan como una forma de comunicarse directamente con los ciudadanos, sin intermediarios que puedan hacerles preguntas comprometidas por ser más conocedores de la situación o tener más información previa, con lo que les es más cómodo colocar su mensaje".

- $\quad$ "Hacen un uso muy limitado de sus perfiles. Los periodistas suelen ser mucho más activos y útiles. Lo más interesante de los perfiles políticos son sus propias impresiones personales, ya que te ofrecen un elemento añadido a la información oficial. Eso sí, los mensajes de los políticos en Twitter tienen mucha influencia porque son seguidos de forma fiel por militantes y dirigentes locales y regionales de sus partidos y cuadros medios, que retuitean y expanden sus mensajes son rapidez y profusión".

- " "Lo usan como medio de expresar sus ideas y llegar a un público más joven".

- "En muchos es un uso inadecuado porque ni siquiera es manejado por ellos mismo, sino por terceras personas".

Aspectos como que son perfiles usados como medios de mera propaganda; que su utilización aún es muy limitada; que no son conscientes de la repercusión y facilidad de propagación de la información allí compartida; que las usan para eliminar intermediarios que pueden tener acceso a mayor información sobre determinados temas; que les sirve de ayuda para dirigirse a un público más joven; o cómo a veces son demasiados atrevidos y generan polémicas innecesarias, han sido los más señalados por estos profesionales.

Unos periodistas a los que también se le pidió aportaran su visión sobre si los políticos usan estas plataformas como un ensayo ante las posibles reacciones de la sociedad ante determinadas situaciones políticas, es decir, ¿estamos frente a una herramienta que les sirve para medir las reacciones de los ciudadanos sobre posibles temas políticos?

De los 6 periodistas, 4 concluyeron que no utilizan estas redes, al menos de momento, para medir las reacciones de los ciudadanos, mientras que 2 de ellos defendieron que a veces si, o que empiezan a hacerlo. De los 4 que afirmaron que no, especificaron que se siguen sirviendo de medios de comunicación afines para lanzar "globos sonda" y no desde sus cuentas al no ser informaciones oficiales, y de encuestas, dejando las redes sociales como un vehículo más para testar el estado de la información pública en general.

Para finalizar y en alusión al uso que los partidos políticos realizan de las redes durante las campañas electorales, quisimos saber la opinión que tenían sobre si los políticos han absorbido el uso de las redes como una nueva forma de conectar con el electorado o por el contrario lo utilizan como un elemento más de campaña.

Las respuestas que obtuvimos son variadas y en varios casos se refiere a que depende del político en cuestión. Sólo 2 de los 6 entrevistados lo consideran un elemento más de campaña y son varios los que afirman que aún no han asimilado su buen uso y desarrollo pleno. Algunas de las respuestas hicieron referencia al uso de perfiles que se abren sólo durante las campañas; la forma en que en función de mayor repercusión mediática las redes se dejan más para un uso propagandístico o emisión de comunicados; así como se hace referencia a que no toda la vida o debate se encuentra en las redes. Algunas respuestas fueron las siguientes:

- $\quad$ "Es un medio más. La comunicación electoral es un conjunto, y ahora las redes sociales son importantes, pero hay vida más allá de esa redes. Vida y debate"

- $\quad$ "Lo utilizan como una nueva forma de conectar directa e inmediatamente con sus bases, militantes, simpatizantes y con los ciudadanos en general. Y no sólo en campaña. En las campañas electorales hay candidatos o políticos que abren un perfil sólo con ese fin: la propaganda electoral, no suelen tener mucho éxito. También hay perfiles electorales oficiales de apoyo a los candidatos. Estos dos tipos de perfiles suelen cerrarse tras las elecciones. En mi opinión, su uso es contraproducente, porque difunden la idea de que sólo les interesan las redes y la comunicación directa con los ciudadanos para que les voten".

- " "Una nueva forma para conectar con su electorado, aunque creo que no han asimilado aún su buen uso"

- $\quad$ "Hay de todo, aunque según van teniendo más repercusión en los medios de comunicación de masas las redes sociales las dejan más para la propaganda o emitir comunicados".

- "En muchos casos sí lo usan para conectar, pero aún está por desarrollar su utilidad plena".

- $\quad$ "En la mayoría de los casos son una forma de multiplicar el mensaje que quieren transmitir y cómo lo quieren transmitir, y en época electoral es la mejor forma que encuentran de transmitir las frases que quieren, sin pasar por el tamiz crítico del periodista". 


\section{Conclusiones}

Llegados a este punto, algunas conclusiones serían, en opinión de estos especialistas del medio, que los políticos tienen un uso limitado y poco aprovechado de las redes sociales; que las utilizan para llegar a un público más joven; que es empleado como medio de propaganda; que suelen estar gestionados por terceras personas; y que pese a ser un medio de fácil propagación de sus mensajes a veces no son conscientes de sus posibilidades y repercusiones.

Para concluir y en vista de los resultados obtenidos a través de estas entrevistas, podríamos definir que el papel del periodista en la información política y electoral está marcado por un uso diario de las redes sociales, que en su mayoría usan desde un punto de vista profesional, tanto para la discusión de textos propios; difusión de noticias de su publicación; subir información de terceros que consideran interesante; consulta e intercambio de información; etc, y que pese a la poca valoración inicial y precaución en cuanto al uso de informaciones obtenidas a través de las redes sociales, la mayoría de estos profesionales del periodismo político han aceptado estos nuevos soportes como una parte complementaria a los medios tradicionales.

De Twitter, los participantes destacaron la rapidez, la concisión, el volumen de información y medición de la repercusión entre los lectores, a la vez que señalaron que es imprescindible saber filtrar que contenidos son erróneos o no rigurosos.

Se ha convertido en una herramienta de utilización diaria en su trabajo, y en cierta medida en una extensión de la redacción, compartiendo en su totalidad información relacionada directamente con su trabajo (además de otras). Tal y como explicamos anteriormente, las informaciones elaboradas en las redacciones van más allá de las ediciones en papel, digitales, o páginas oficiales en redes sociales, y se adentran en los perfiles personales de quienes forman parte de la plantilla y publicitan su trabajo y el de sus compañeros.

\section{Bibliografía}

CANEL, M. J. Comunicación Política: Una guía para su estudio y práctica. Tecnos, 2006

CAMPOS DOMÍNGUEZ, Eva. BERROCAL GONZALO, Salomé. (coord.) La investigación en periodismo político en el entorno de los nuevos medios Sociedad Española de Periodística, 2012

CAPDEVILLA DOMÍNGUEZ, David. Democracia 2.0: la política se introduce en las redes sociales Pensar la publicidad, 2009, vol III, n², $31-48$

CASERO-RIPOLLÉS, Andreu. YESTE, Elena. La comunicación política hoy: entre nuevos medios y viejas lógicas. Trípodos, n 34, 9-12

CASERO-RIPOLLÉS, Andreu. Periodismo político en España: concepciones, tensiones y elecciones. CAL, Cuadernos Artesanos de Latina $n^{0} 33$

CASERO-RIPOLLÉS, Andreu, GUTIÉRREZ-RUBÍ, Antoni. ¿Un cambio de paradigma? Democracia y nuevos medios digitales Telos, n 98. 44-47

COLOMÉ, Gabriel. Política y medios de comunicación: una aproximación teórica Working papee n. 91 Barcelona 1994

DADER, J. L. Periodismo de precisión: Vía Socio informática de descubrir noticias,1997

KEANE, JOHN, FEENSTRA, Ramón A. Democracia monitorizada en España. Nuevas formas de participación política en el marco de la era digital. Telos $n^{\circ}$ 98. 48-57

MAAREK, P. J. Marketing político y comunicación: claves para una buena información política Barcelona: Paidós, 1997

MARTÍNEZ NICOLÁS, Manuel, HUMANES, Mª Luisa, SAPERAS LAPIEDRA, Enric La mediatización de la política en el periodismo español. Análisis longitudinal de la información política en la prensa de referencia (1980-2010) Trípodos, $n^{0} 34$.Barcelona 2014, 41-59

MUÑOZ ALONSO, A. La comunicación política en España. Informe anual de la profesión periodística, 2008, pág, 37-42

ORTEGA, Félix. Una simbiosis compleja. Políticos y periodistas. Segunda Época n 54.2003

PANIAGUA, F. J. Comunicación política y elecciones: nuevas prácticas en el escenario español, 2004

RODRIGUEZ ANDRÉS, Roberto, UREÑA UCEDA, Daniel. Diez razones para el uso de Twitter como herramienta en la comunicación política y electoral.

SAMPREDO BLANCO, Víctor F. Comunicación Política: nuevos medios, nuevas audiencias, nuevos problemas. Telos.

VASALLO DE LOPES, M.I. La investigación de la comunicación: cuestiones epistemológicas, teóricas y metodológicas Revista académica de la Federación Latinoamericana de comunicación social

Otras fuentes

Join the conversation: How spanish journalism are using Twitter. LABÁPART. Universidad Carlos III Madrid. 
http://www.pilarcarrera.es/jointheconversation.html

Reuters Institute Digital News Report 2014: Spain

http://www.unav.edu/web/digital-studies/news

V Estudio anual de redes sociales del IAB (Interactive Advertisinf Bureau)

http://www.iabspain.net/investigacion/

\section{Cómo citar este artículo en bibliografías - How to cite this article in bibliographies / references:}

PARRA-GÓMEZ, L. (2016): "El papel del periodista en la información política electoral y uso de Twitter en la difusión de contenidos". En Revista de la Asociación Española de Investigación de la Comunicación, vol. 3, número 5, pp. 64-74. 